\title{
Space-time structure of new physics with polarized beams at the linear collider
}

\author{
B. Ananthanarayan ${ }^{a}$, Saurabh D. Rindani ${ }^{b}$ \\ ${ }^{a}$ Centre for High Energy Physics, Indian Institute of Science \\ Bangalore 560 012, India \\ ${ }^{b}$ Theory Group, Physical Research Laboratory \\ Navrangpura, Ahmedabad 380 009, India
}

\begin{abstract}
We approach the issue of the discovery of new physics at high energies associated with the proposed International Linear Collider in the presence of longitudinal as well as transverse electron and positron beam polarization. We determine the beam polarization dependence and the angular distribution of a particle of arbitrary spin in a one-particle inclusive final state produced in $e^{+} e^{-}$collisions through the interference of $\gamma$ or $Z$ amplitude with the amplitude from new interactions having arbitrary space-time structure. We thus extend the results of Dass and Ross proposed at the time of the discovery of neutral currents, to beyond the standard model currents. We also extend the case of $e^{+} e^{-}$annihilation in the $s$-channel to the production of bosons due to $t$ - and $u$-channel processes. Our work provides an approach to model-independent determination of the space-time structure of beyond the standard model interactions. We briefly discuss applications of the framework to popular extensions of the standard model, and demonstrate that our framework is general enough to account for certain results in the minimal supersymmetric standard model.
\end{abstract}

\section{Introduction}

The possibility of the International Linear Collider (ILC) that will collide electrons and positrons at a large centre of mass energy, of $\sqrt{s}=500 \mathrm{GeV}$, or even $800 \mathrm{GeV}$ is rapidly becoming a reality [1]. It is also likely that the beams can be significantly polarized and there has been a great international effort at exploring the physics possibilities with such a facility [2]. It has been recently shown that the availability of transverse polarization could open up the possibility of observing $\mathrm{CP}$ violation in $t \bar{t}$ production due to the possibility of beyond the standard model (BSM) interactions due to scalar and tensor type interactions 3 . On the other hand, it has been shown that the availability of longitudinal polarization can significantly improve the sensitivity to $\mathrm{CP}$-violating dipole moments in this process [4, and 
analogously for $\tau^{+} \tau^{-}$production [5] at linear collider energies, following ideas proposed earlier in the context of the tau-charm factories [6]. In the recent past, we have also pointed out that BSM interactions could lead to CP-violating anomalous triple-gauge boson vertices, the sensitivity to which could be enhanced by the availability of longitudinal beam polarization [7] and transverse polarization [8]. It has been further pointed out that the most general contact interactions could also be explored exhaustively by the availability of each type of polarization [9, 10. In the specific context of transversely polarized beams, some works of interest are [11, 12] These investigations have revealed that it is fruitful to investigate in as great generality as possible the physics that can be explored at the ILC with polarization.

An analogous situation arose in the in the 1970's at the time the neutral currents had just been discovered. Dass and Ross [13, 14] considered the possibility of establishing the spacetime structure of the neutral currents by considering the interference of the well-known QED process for electron-positron annihilation with the then 'new' physics currents to leading order. Allowing for all possible transformation properties of the neutral current under the Lorentz transformation, it was possible to ask for how the signatures would differ in the correlations amongst the momenta of the electron, positron and one of the reaction products, and the spins of the electron and positron, allowing for all possible types of polarization of the initial state particles. The result was a table for all the possible correlations, along with their $\mathrm{C}$ and $\mathrm{P}$ properties, which provides a standard reference for establishing what kind of interaction, viz. scalar, pseudoscalar, vector, axial vector and tensor could possibly interfere with the QED amplitude and produce possibly CP-violating signals.

Today, one could use the effective current induced by the BSM interaction and read off the correlation that would result from its interference with the QED amplitude. However, this would be incomplete as the standard model (SM) amplitude results from the sum of the QED as well as the neutral current amplitude due to the exchange of a virtual $Z$, whose contribution is comparable to the QED amplitude at LC energies. It may be noted that the situation with the neutral current is more complicated than the QED case as the $Z$ has a vector as well as an axial-vector coupling to the electron. It should be pointed out here that our results are rather general; as we will show, angular correlations involving sparticles in the minimal supersymmetric standard model (MSSM) can also be accounted for in this framework.

Indeed, in the case of $t \bar{t}$ it was possible to guess that there would be no CP-violating observable with only transversely polarized beams if all BSM interactions were of the vector or axial-vector type, which was borne out by explicit computations, with amplitudes for BSM physics given in an effective Lagrangian framework [3], even though a general discussion of the Dass and Ross type was not available when both QED and $Z$ exchange contributions are included.

In this work, we will now provide such a general discussion and provide an explicit table for the correlations of interest in the presence of both vector and axial-vector couplings of the intermediate vector boson. This has the additional advantage of not having to appeal to any effective low-energy theory or an effective Lagrangian framework. We will, however, 
point out the connections of the present work to those presented in ref. [3]. The framework employed therein was the one presented in ref. [15], which is a restrictive one, where a special class of couplings is considered.

Since we restrict ourselves to the the measurement of the energy-momentum of a single particle in the final state, we exclude the possibility that the spin of the final state particle is measured. The question we are asking is how far can we go in the analysis of the new physics using only the energy and momentum of a single final-state particle, but equipped with polarized $e^{-}$and $e^{+}$beams. It should be noted that we implicitly include the case when the single final-state particle studied could be the decay product of an unstable particle.

Considerations of this type cannot applied directly to the situation when the SM contributions to a process come from $t$ - and $u$-channel contributions. However, use can be made of our results with appropriate modifications. We provide a discussion on this subject.

In Sec. 2 we describe the calculation of single-particle angular distributions arising from the interference of $s$-channel exchange SM terms with the BSM terms of arbitrary space-time structure. In Sec. 3, we discuss to what extent form factors introduced in Sec. 2 may be determined from the angular distributions. Sec. 4 is devoted to a discussion of the CP properties of the various correlations. A discussion of SM processes with $t$ - and $u$-channel exchanges is given in Sec. 5. Some applications of our formalism are presented in Sec. 6. followed by our conclusions in Sec. 7 .

\section{Computation of correlations}

We consider the one-particle inclusive process

$$
e^{-}\left(p_{-}\right)+e^{+}\left(p_{+}\right) \rightarrow H(p)+X,
$$

where $H$ is a final state particle, whose momentum $p$ is measured, but not the spin, and $X$ is an inclusive state. The process is assumed to occur through an $s$-channel exchange of a photon and a $Z$ in the SM, and through a new current whose coupling to $e^{+} e^{-}$can be of the type $V, A$, or $S, P$, or $T$.

Since we will deal with a general case without specifying the nature or couplings of $H$, we do not attempt to write the amplitude for the process (11). We will only obtain the general form, in each case of the new coupling, of the contribution to the angular distribution of $H$ from the interference of the SM amplitude with the new physics amplitude.

Following Dass and Ross [13, 14, we calculate the relevant factor in the interference between the standard model currents with the BSM currents as

$$
\operatorname{Tr}\left[\left(1-\gamma_{5} h_{+}+\gamma_{5} \phi_{+}\right) \not p_{+} \gamma_{\mu}\left(g_{V}^{e}-g_{A}^{e} \gamma_{5}\right)\left(1+\gamma_{5} h_{-}+\gamma_{5} \phi_{-}\right) \not p_{-} \Gamma_{i}\right] H^{i \mu}
$$

Here $g_{V}^{e}, g_{A}^{e}$ are the vector and axial-vector couplings of the photon or $Z$ to the electron current, and $\Gamma_{i}$ is the corresponding coupling to the new physics current, $p_{ \pm}$are the fourmomenta of $e^{ \pm}, h_{ \pm}$are the helicities (in units of $\frac{1}{2}$ ) of $e^{ \pm}$, and $s_{ \pm}$are respectively their 
transverse polarizations. For ease of comparison, we have sought to stay with the notation for refs. [13, 14, with some exceptions which we spell out when necessary. We should of course add the contributions coming from photon exchange and $Z$ exchange, with the appropriate propagator factors. However, we give here the results for $Z$ exchange, from which the case of photon can be deduced as a special case. The tensor $H^{i \mu}$ stands for the interference between the couplings of the final state to the SM current and the new physics current, summed over final-state polarizations, and over the phase space of the unobserved particles $X$. It is only a function of the the momenta $q=p_{-}+p_{+}$and $p$. The implied summation over $i$ corresponds to a sum over the forms $V, A, S, P, T$, together with any Lorentz indices that these may entail. We will continue to use for the tensor $H^{i \mu}$ the term "hadronic tensor", which is of historical origin, and whose use was relevant to the case when the final states considered were hadronic.

We now determine the forms of the matrices $\Gamma_{i}$ and the tensors $H^{i \mu}$ in the various cases, using only Lorentz covariance properties. Our additional currents are as in refs. 13, 14, except for the sign of $g_{A}$ in the following. We explicitly note that in our convention is $\epsilon_{0123}=+1$. Our results for the $\epsilon$ terms differ from those in refs. [13, 14] by a sign, which we attribute to a different sign convention for the $\epsilon$ symbol, a convention that is not explicitly spelt out in [13, 14]. We set the electron mass to zero. Consider now the three cases:

1. Scalar and Pseudoscalar case:

In this case, there is no free Lorentz index for the leptonic coupling. Consequently, we can write it as

$$
\Gamma=g_{S}+i g_{P} \gamma_{5}
$$

The tensor $H^{i \mu}$ for this case has only one index, viz., $\mu$. Hence the most general form for $H$ is

$$
H_{\mu}^{S}=F\left(q^{2}, p \cdot q\right) p_{\mu},
$$

where $\mathrm{F}$ is a function of the Lorentz-invariant quantities $q^{2}$ and $p \cdot q$.

\section{Vector and Axial-Vector case:}

The leptonic coupling for this case can be written as

$$
\Gamma_{\mu}=\gamma_{\mu}\left(g_{V}-g_{A} \gamma_{5}\right)
$$

Note that we differ from Dass and Ross [13, 14 in the sign of the $g_{A}$ term. The tensor $H$ for this case has two indices, and can be written as

$$
H_{\mu \nu}^{V}=-g_{\mu \nu} W_{1}\left(q^{2}, p \cdot q\right)+p_{\mu} p_{\nu} W_{2}\left(q^{2}, p \cdot q\right)+\epsilon_{\mu \nu \alpha \beta} q^{\alpha} p^{\beta} W_{3}\left(q^{2}, p \cdot q\right),
$$

where now there are three invariant functions, $W_{1}, W_{2}, W_{3}$.

\section{Tensor case:}

In the tensor case, the leptonic coupling is

$$
\Gamma_{\mu \nu}=g_{T} \sigma_{\mu \nu}
$$




\begin{tabular}{||c|c|c|c||}
\hline Term & Correlation & $\mathrm{P}$ & $\mathrm{C}$ \\
\hline \hline $\operatorname{Im}\left(g_{P} F\right)$ & $-2 E^{2}\left(\vec{s}_{+}-\vec{s}_{-}\right) \cdot \vec{p}$ & - & - \\
$\operatorname{Im}\left(g_{S} F\right)$ & $2 E\left[\vec{K} \cdot\left(\vec{s}_{+}+\vec{s}_{-}\right) \times \vec{p}\right]$ & + & - \\
$\operatorname{Re}\left(g_{S} F\right)$ & $2 E^{2} \vec{p} \cdot\left(h_{+} \vec{s}_{-}-h_{-} \vec{s}_{+}\right)$ & + & - \\
$\operatorname{Re}\left(g_{P} F\right)$ & $-2 E\left[\vec{K} \cdot\left(h_{+} \vec{s}_{-}+h_{-} \vec{s}_{+}\right] \times \vec{p}\right)$ & - & - \\
\hline
\end{tabular}

Table 1: List of $S, P$ correlations for $g_{V}^{e}$

\begin{tabular}{||c|c|c|c||}
\hline Term & Correlation & $\mathrm{P}$ & $\mathrm{C}$ \\
\hline \hline $\operatorname{Im}\left(g_{P} F\right)$ & $2 E^{2}\left(h_{+} \vec{s}_{-}+h_{-} \vec{s}_{+}\right) \cdot \vec{p}$ & + & + \\
$\operatorname{Im}\left(g_{S} F\right)$ & $2 E\left[\vec{K} \cdot\left(h_{+} \vec{s}_{-}-h_{-} \vec{s}_{+}\right) \times \vec{p}\right]$ & - & + \\
$\operatorname{Re}\left(g_{S} F\right)$ & $2 E^{2} \vec{p} \cdot\left(\vec{s}_{+}+\vec{s}_{-}\right)$ & - & + \\
$\operatorname{Re}\left(g_{P} F\right)$ & $2 E\left[\vec{K} \cdot\left(\vec{s}_{+}-\vec{s}_{-}\right) \times \vec{p}\right]$ & + & + \\
\hline
\end{tabular}

Table 2: List of $S, P$ correlations for $g_{A}^{e}$

The tensor $H$ for this case can be written in terms of the four invariant functions $F_{1}, F_{2}, P F_{1}, P F_{2}$ as

$$
\begin{aligned}
H_{\mu \rho \tau}^{T}= & \left(q_{\rho} p_{\tau}-q_{\tau} p_{\rho}\right) p_{\mu} F_{1}\left(q^{2}, p \cdot q\right)+\left(g_{\rho \mu} p_{\tau}-g_{\tau \mu} p_{\rho}\right) F_{2}\left(q^{2}, p \cdot q\right) \\
& +\epsilon_{\rho \tau \alpha \beta} p^{\alpha} q^{\beta} p_{\mu} P F_{1}\left(q^{2}, p \cdot q\right)+\epsilon_{\rho \tau \mu \alpha} p^{\alpha} P F_{2}\left(q^{2}, p \cdot q\right) .
\end{aligned}
$$

We next substitute the leptonic vertices $\Gamma$ and the respective tensors $H^{i}$ in (2), and evaluate the trace in each case. We present the results in Tables 1-6, with $\vec{K} \equiv\left(\vec{p}_{-}-\vec{p}_{+}\right) / 2=$ $E \hat{z}$, where $\hat{z}$ is a unit vector in the z-direction, $E$ is the beam energy, and $\vec{s}_{ \pm}$lie in the $\mathrm{x}-\mathrm{y}$ plane.

In Tables 1, 3 and 5 the results presented in ref. [13, 14, are reproduced in our convention, since these are for the case of $g_{V}^{e}$ alone, which was the case considered by these authors for the interference of QED amplitudes with physics due to the then undetermined amplitude of the neutral current due to $Z$. The corresponding tables for $g_{A}^{e}$ are now presented in Tables 2, 4 and 6 , for cases of scalar-pseudoscalar, vector-axial-vector and tensor couplings respectively.

In Tables 1-6 are also given the charge conjugation $\mathrm{C}$ and parity $\mathrm{P}$ properties of the various correlations, under the assumption that the final-state particle observed is self-conjugate, viz., $H=\bar{H}$. If it is not self-conjugate, then the $\mathrm{C}$ factor given in the tables would apply to the sum of the cross sections for production of $H$ and $\bar{H}$. The difference of these cross sections would take a $\mathrm{C}$ factor of the opposite sign. 


\begin{tabular}{||c|c|c|c||}
\hline Term & Correlation & $\mathrm{P}$ & $\mathrm{C}$ \\
\hline \hline $\operatorname{Re}\left(g_{V} W_{1}\right)$ & $-4 E^{2}\left(h_{+} h_{-}-1\right)$ & + & + \\
$\operatorname{Re}\left(g_{A} W_{1}\right)$ & $4 E^{2}\left(h_{+}-h_{-}\right)$ & - & - \\
$\operatorname{Re}\left(g_{V} W_{2}\right)$ & $-2\left[2 E^{2} \vec{p} \cdot \overrightarrow{s_{-}} \vec{p} \cdot \vec{s}_{+}+\left(\vec{K} \cdot \vec{K} \vec{p} \cdot \vec{p}-(\vec{p} \cdot \vec{K})^{2}\right)\left(h_{+} h_{-}-1-\vec{s}_{+} \cdot \vec{s}_{-}\right)\right]$ & + & + \\
$\operatorname{Re}\left(g_{A} W_{2}\right)$ & $2\left(\vec{K} \cdot \vec{K} \vec{p} \cdot \vec{p}-(\vec{p} \cdot \vec{K})^{2}\right)\left(h_{+}-h_{-}\right)$ & - & - \\
$\operatorname{Im}\left(g_{V} W_{3}\right)$ & $8 E^{2}(\vec{p} \cdot \vec{K})\left(h_{+}-h_{-}\right)$ & - & + \\
$\operatorname{Im}\left(g_{A} W_{3}\right)$ & $-8 E^{2}(\vec{p} \cdot \vec{K})\left(h_{+} h_{-}-1\right)$ & + & - \\
$\operatorname{Im}\left(g_{A} W_{2}\right)$ & $2 E\left(\vec{p} \cdot \vec{s}_{+}\left[\vec{K} \cdot \overrightarrow{s_{-}} \times \vec{p}\right]+\vec{p} \cdot \vec{s}_{-}\left[\vec{K} \cdot \vec{s}_{+} \times \vec{p}\right]\right)$ & - & - \\
\hline
\end{tabular}

Table 3: List of $V, A$ correlations for $g_{V}^{e}$

\section{Determination of form factors from correlations}

For simplicity and for the purposes of this section, we do not consider simultaneous presence of both longitudinal and transverse polarizations. Thus, we will discuss the possibilities of unpolarized beams, pure longitudinal polarization and pure transverse polarization. We would like to investigate how many of the independent form factors can be determined by the independent angular correlations available.

By examining the tables it can be seen that in the absence of polarization, the only correlations which survive are in the case of $V, A$ interactions. Even in that case, it is only possible to get information on the quantities $\operatorname{Re}\left(g_{V} W_{1}\right), \operatorname{Re}\left(g_{V} W_{2}\right)$ and $\operatorname{Im}\left(g_{A} W_{3}\right)$ in association with $g_{V}^{e}$, and $\operatorname{Re}\left(g_{A} W_{1}\right), \operatorname{Re}\left(g_{A} W_{2}\right)$ and $\operatorname{Im}\left(g_{V} W_{3}\right)$ in association with $g_{A}^{e}$. However, they give only three independent angular distributions, and as such, only 3 combinations of these 6 quantities can be determined in the absence of polarization.

In the presence of longitudinal polarization alone, it is possible to get information on 3 more combinations of form factors, proportional to $\left(h_{+}-h_{-}\right)$, using either $e^{-}$or $e^{+}$ polarization in case of $V$ and $A$ couplings. It is not necessary to have both $e^{-}$and $e^{+}$beams polarized.

It is clear why only $V$ and $A$ couplings can contribute in the absence of polarization, or in the presence of only longitudinal polarization. We are concentrating on interference between the $V, A$ SM contribution ${ }^{1}$ and the new physics contribution. Hence the terms which survive should correspond to the same combination of $e^{-}$and $e^{+}$helicities. Since $V, A$ amplitudes have a different helicity structure as compared to the amplitudes coming from the $S, P, T$ structure from BSM, they do not interfere.

The case with transverse polarization is somewhat different. Since transverse polarization corresponds to a linear combination of two different helicities, it is possible for interference terms of different helicity structures to survive.

\footnotetext{
${ }^{1}$ Our framework being sufficiently general may readily be generalized to $V, A$ contributions in other theories, e.g., the MSSM. For a discussion of certain results therein, see Sec. 5, 6 .
} 


\begin{tabular}{||c|c|c|c||}
\hline Term & Correlation & $\mathrm{P}$ & $\mathrm{C}$ \\
\hline \hline $\operatorname{Re}\left(g_{V} W_{1}\right)$ & $4 E^{2}\left(h_{+}-h_{-}\right)$ & - & - \\
$\operatorname{Re}\left(g_{A} W_{1}\right)$ & $-4 E^{2}\left(h_{+} h_{-}-1\right)$ & + & + \\
$\operatorname{Re}\left(g_{V} W_{2}\right)$ & $2\left(\vec{K} \cdot \vec{K} \vec{p} \cdot \vec{p}-(\vec{p} \cdot \vec{K})^{2}\right)\left(h_{+}-h_{-}\right)$ & - & - \\
$\operatorname{Re}\left(g_{A} W_{2}\right)$ & $-2\left[-2 E^{2} \vec{p} \cdot \vec{s}_{-} \vec{p} \cdot \vec{s}_{+}+\left(\vec{K} \cdot \vec{K} \vec{p} \cdot \vec{p}-(\vec{p} \cdot \vec{K})^{2}\right)\left(h_{+} h_{-}-1+\vec{s}_{+} \cdot \vec{s}_{-}\right)\right]$ & + & + \\
$\operatorname{Im}\left(g_{V} W_{3}\right)$ & $-8 E^{2}(\vec{p} \cdot \vec{K})\left(h_{+} h_{-}-1\right)$ & + & - \\
$\operatorname{Im}\left(g_{A} W_{3}\right)$ & $8 E^{2}(\vec{p} \cdot \vec{K})\left(h_{+}-h_{-}\right)$ & - & + \\
$\operatorname{Im}\left(g_{V} W_{2}\right)$ & $-2 E\left(\vec{p} \cdot \vec{s}_{+}\left[\vec{K} \cdot \overrightarrow{s_{-}} \times \vec{p}\right]+\vec{p} \cdot \vec{s}_{-}\left[\vec{K} \cdot \vec{s}_{+} \times \vec{p}\right]\right)$ & - & - \\
\hline
\end{tabular}

Table 4: List of $V, A$ correlations for $g_{A}^{e}$

\begin{tabular}{||c|c|c|c||}
\hline Term & Correlation & $\mathrm{P}$ & $\mathrm{C}$ \\
\hline \hline $\operatorname{Im}\left(g_{T} F_{1}\right)$ & $-8 E^{2} \vec{p} \cdot \vec{K}\left[\vec{p} \cdot\left(h_{+} \vec{s}_{-}-h_{-} \vec{s}_{+}\right)\right]$ & + & + \\
$\operatorname{Im}\left(g_{T} F_{2}\right)$ & $4 E^{2} \vec{p} \cdot\left(h_{+} \vec{s}_{-}+h_{-} \vec{s}_{+}\right)$ & + & + \\
$\operatorname{Im}\left(g_{T} P F_{1}\right)$ & $8 E \vec{p} \cdot \vec{K}\left[\vec{K} \cdot\left(h_{+} \vec{s}_{-}+h_{-} \vec{s}_{+}\right) \times \vec{p}\right]$ & - & + \\
$\operatorname{Im}\left(g_{T} P F_{2}\right)$ & $4 E\left[\vec{K} \cdot\left(h_{+} \vec{s}_{-}-h_{-} \vec{s}_{+}\right) \times \vec{p}\right]$ & - & + \\
$\operatorname{Re}\left(g_{T} F_{1}\right)$ & $8 E \vec{p} \cdot \vec{K}\left[\vec{K} \cdot\left(\vec{s}_{+}+\vec{s}_{-}\right) \times \vec{p}\right]$ & + & + \\
$\operatorname{Re}\left(g_{T} F_{2}\right)$ & $4 E\left[\vec{K} \cdot\left(\vec{s}_{+}-\vec{s}_{-}\right) \times \vec{p}\right]$ & + & + \\
$\operatorname{Re}\left(g_{T} P F_{1}\right)$ & $-8 E^{2} \vec{p} \cdot \vec{K}\left[\vec{p} \cdot\left(\vec{s}_{+}-\vec{s}_{-}\right)\right]$ & - & + \\
$\operatorname{Re}\left(g_{T} P F_{2}\right)$ & $4 E^{2} \vec{p} \cdot\left(\vec{s}_{+}+\vec{s}_{-}\right)$ & - & + \\
\hline
\end{tabular}

Table 5: List of $T$ correlations for $g_{V}^{e}$

With only transverse polarization, it can be seen from the tables that one can get information on all 4 combinations of $S, P$ type couplings, 3 combinations of $V, A$ type couplings involving only $W_{2}$, and 8 combinations of $T$ type couplings. Of course, if $S, P$ and $T$ couplings are present simultaneously, then only an overall total of 8 combinations can be determined.

In the case of $S, P$ and $T$ couplings, it is sufficient to have either $e^{-}$or $e^{+}$beams polarized. In case of $V, A$, both beams have to be polarized, or the effect vanishes. It is interesting to note that all the correlations in the latter case are symmetric under the interchange of $\vec{s}_{+}$ and $\vec{s}_{-}$.

An inspection of the momentum and spin correlations of Tables 5 and 6 reveals that apart from overall multiplicative factors including those of energy and scalar products of momenta, the momentum and spin correlations are precisely those that are found in Tables 1 and 2 .

The counting of the number of independent correlations for the vector and axial-vector cases turns out to be subtle. First consider having only non-zero $g_{V}^{e}$. The number of independent form factors that contribute to the correlations is 7 , and the number of correlations is 


\begin{tabular}{||c|c|c|c||}
\hline Term & Correlation & $\mathrm{P}$ & $\mathrm{C}$ \\
\hline \hline $\operatorname{Im}\left(g_{T} F_{1}\right)$ & $-8 E^{2} \vec{p} \cdot \vec{K}\left[\vec{p} \cdot\left(\vec{s}_{+}+\vec{s}_{-}\right)\right]$ & - & - \\
$\operatorname{Im}\left(g_{T} F_{2}\right)$ & $-4 E^{2} \vec{p} \cdot\left(\vec{s}_{+}-\vec{s}_{-}\right)$ & - & - \\
$\operatorname{Im}\left(g_{T} P F_{1}\right)$ & $-8 E \vec{p} \cdot \vec{K}\left[\vec{K} \cdot\left(\vec{s}_{+}-\vec{s}_{-}\right) \times \vec{p}\right]$ & + & - \\
$\operatorname{Im}\left(g_{T} P F_{2}\right)$ & $4 E\left[\vec{K} \cdot\left(\vec{s}_{+}+\vec{s}_{-}\right) \times \vec{p}\right]$ & + & - \\
$\operatorname{Re}\left(g_{T} F_{1}\right)$ & $8 E \vec{p} \cdot \vec{K}\left[\vec{K} \cdot\left(h_{+} \vec{s}_{-}-h_{-} \vec{s}_{+}\right) \times \vec{p}\right]$ & - & - \\
$\operatorname{Re}\left(g_{T} F_{2}\right)$ & $-4 E\left[\vec{K} \cdot\left(h_{+} \vec{s}_{-}+h_{-} \vec{s}_{+}\right) \times \vec{p}\right]$ & - & - \\
$\operatorname{Re}\left(g_{T} P F_{1}\right)$ & $8 E^{2} \vec{p} \cdot \vec{K}\left[\vec{p} \cdot\left(h_{+} \vec{s}_{-}+h_{-} \vec{s}_{+}\right)\right]$ & + & - \\
$\operatorname{Re}\left(g_{T} P F_{2}\right)$ & $4 E^{2} \vec{p} \cdot\left(h_{+} \vec{s}_{-}-h_{-} \vec{s}_{+}\right)$ & + & - \\
\hline
\end{tabular}

Table 6: List of $T$ correlations for $g_{A}^{e}$

7. When we consider the case when only $g_{A}^{e}$ is non-zero, there are again 7 independent form factors, of which 6 are common to the previous list. More explicitly, $\operatorname{Im}\left(g_{A} W_{2}\right)$ contributes to the correlations only via $g_{V}^{e}$, while $\operatorname{Im}\left(g_{V} W_{2}\right)$ contributes to the correlations only via $g_{A}^{e}$, noting however that the resulting correlation is exactly the same apart from the sign. Indeed, the momentum and spin correlations also have the structure of 6 being in common and 1 each from each of the two cases above; the correlation due to $\operatorname{Re}\left(g_{V} W_{2}\right)$ via $g_{V}^{e}$ differs from the correlation resulting via $g_{A}^{e}$. Thus, although the independent form factors and the corresponding momentum and spin correlations are not in one to one correspondence, each set adds up separately to 8 . The total number of independent form factors is 12 , and even if both longitudinal and transverse polarizations are used, 4 of these, viz., $\operatorname{Im}\left(g_{V} W_{1}\right), \operatorname{Im}\left(g_{A} W_{1}\right)$, $\operatorname{Re}\left(g_{V} W_{3}\right)$ and $\operatorname{Re}\left(g_{A} W_{3}\right)$ do not enter the distributions, and remain undetermined.

\section{CP properties of correlations}

We might like to use the behaviour of the differential cross section to construct asymmetries which can test symmetry properties like CP. Tables 1-6 may be employed to make some predictions for what to expect.

There are some general deductions we can make in the special case when the final-state is a two particle state. Within that, we consider two possibilities:

Case 1: $H=\bar{H}$ The simplest case to consider is when $H$ is self-conjugate, i.e., $H=\bar{H}$.

In the absence of polarization, of the 3 independent angular distributions, Tables 3 and 4 show that only one, viz., $\vec{p} \cdot \vec{K}=E|\vec{p}| \cos \theta$, where $\theta$ is the angle made by $\vec{p}$ with the $e^{-}$beam direction, is CP violating. It accompanies $\operatorname{Im}\left(g_{V} W_{3}\right)$ or $\operatorname{Im}\left(g_{A} W_{3}\right)$, and needs the absorptive part of some amplitude to be nonzero, consistent with the CPT theorem.

With longitudinal polarization alone, of the three additional combinations possible, one is CP violating, again proportional to $\vec{p} \cdot \vec{K}=E|\vec{p}| \cos \theta$. 
It may be deduced from the tables that for the case of only transverse polarization, CPviolating correlations arise in the case of the terms $g_{V}^{e} \operatorname{Im}\left(g_{S} F\right), g_{A}^{e} \operatorname{Re}\left(g_{S} F\right), g_{V}^{e} \operatorname{Re}\left(g_{T} P F_{1}\right)$, $g_{V}^{e} \operatorname{Re}\left(g_{T} P F_{2}\right), g_{A}^{e} \operatorname{Im}\left(g_{T} P F_{1}\right)$ and $g_{A}^{e} \operatorname{Im}\left(g_{T} P F_{2}\right)$. As in the case of QED, even in the electroweak theory, the interference of new currents cannot lead to a $\mathrm{CP}$-violating correlation due to vector and axial-vector type couplings. With transverse polarization, one can probe $\mathrm{CP}$ violation only due to new physics of the $S, P$ and $T$ type.

Case $2: H \neq \bar{H}$

As mentioned earlier, in this case, the $\mathrm{C}$ properties in the tables refer to the sum

$$
\Delta \sigma^{+}=\Delta \sigma+\Delta \bar{\sigma}
$$

where $\Delta \sigma$ and $\Delta \bar{\sigma}$ are partial cross sections corresponding respectively to $H$ and $\bar{H}$ production. The difference of these,

$$
\Delta \sigma^{-}=\Delta \sigma-\Delta \bar{\sigma}
$$

will have the opposite $\mathrm{C}$ property.

We now consider two special cases: when the final state consists of a pair of conjugate particles $H \bar{H}$, and when it consists of two particles $H \bar{H}^{\prime}$, where $H^{\prime} \neq H$.

Case $2 \mathrm{a}: X \equiv \bar{H}$

In this case, $\Delta \bar{\sigma}$ is obtained from $\Delta \sigma$ by simply the reversal of the sign of $\vec{p}$. In this case, the correlations which are odd in $\vec{p}$ vanish in $\Delta \sigma^{+}$. They survive in $\Delta \sigma^{-}$, but have the opposite $\mathrm{C}$ property as compared to the one shown in the corresponding entry in the relevant table. The correlations which are even in $\vec{p}$, on the other hand, vanish in $\Delta \sigma^{-}$, but survive in $\Delta \sigma^{+}$, and have the same $\mathrm{C}$ property as the one shown in the table.

We can deduce the following for the case of the $H \bar{H}$ final state. Since the only correlations shown as CP odd in Tables 3 and 4 are linear in $\vec{p}$, they do not survive in $\Delta \sigma^{+}$. Hence there are no CP-odd correlations in the $V, A$ case. This statement is true regardless of whether there is polarization or not. In the $S, P$ case, all terms are linear in $\vec{p}$. Hence they all vanish in $\Delta \sigma^{+}$. They do survive in $\Delta \sigma^{-}$, and have CP properties opposite of those shown in Tables 1,2. Thus, considering only transverse polarizations, the terms corresponding to $g_{V}^{e} \operatorname{Im}\left(g_{P} F\right)$ and $g_{A}^{e} \operatorname{Re}\left(g_{P} F\right)$ are the ones odd under CP, whereas the terms corresponding to $g_{V}^{e} \operatorname{Im}\left(g_{S} F\right)$ and $g_{A}^{e} \operatorname{Re}\left(g_{S} F\right)$ are even under CP. Finally, in the $T$ case, one can check that the terms corresponding to $g_{V}^{e} \operatorname{Re}\left(g_{T} F_{2}\right) g_{V}^{e} \operatorname{Re}\left(g_{T} P F_{1}\right), g_{A}^{e} \operatorname{Im}\left(g_{T} F_{2}\right)$ and $g_{A}^{e} \operatorname{Im}\left(g_{T} P F_{1}\right)$ are CP odd and the remaining, viz., $g_{V}^{e} \operatorname{Re}\left(g_{T} F_{1}\right) g_{V}^{e} \operatorname{Re}\left(g_{T} P F_{2}\right), g_{A}^{e} \operatorname{Im}\left(g_{T} F_{1}\right)$ and $g_{A}^{e} \operatorname{Im}\left(g_{T} P F_{2}\right)$ are CP even. It is interesting to note that in all the above, the CP-odd cases correspond to a combination $\left(\vec{s}_{+}-\vec{s}_{-}\right)$of the $e^{+}$and $e^{-}$spin vectors. The reason is that since in the cm frame the momentum vectors of $H$ and $\bar{H}$ are equal and opposite, $\vec{p}$ is equivalent to $\left(\vec{p}_{H}-\vec{p}_{\bar{H}}\right)$, and hence even under CP, as is $\vec{K}$. The only quantity odd under CP is $\left(\vec{s}_{+}-\vec{s}_{-}\right)$, and hence necessary for the term to be CP odd.

We can also consider the possibility that there are no loop effects or final-state interactions. In that case, the $g_{i},(i=S, P, V, A, T)$ are all real, and the form factors $F_{1}$ and 
$W_{3}$ are purely imaginary, the rest of the form factors being real. Then, the only CP-odd contributions are $g_{A}^{e} \operatorname{Re}\left(g_{P} F\right)$ in the $S, P$ case, and the terms corresponding to $g_{V}^{e} \operatorname{Re}\left(g_{T} F_{2}\right)$ and $g_{A}^{e} \operatorname{Im}\left(g_{T} P F_{1}\right)$ in the tensor case.

Case $2 \mathrm{~b}: X \equiv \bar{H}^{\prime}, H \neq H^{\prime}$

We now consider the possibility that the final-state is a two-particle state, of the form $H \bar{H}^{\prime}$, where $H^{\prime}$ is not the same as $H$. In such a case, we cannot rule out a priori either the CP-even or the CP-odd combinations. However, we can make definite statements about $\mathrm{CP}$-odd terms only in the special case that we restrict ourselves to tree-level contributions, and there are no loop contributions or final-state interactions. The simplification in this case is that the effective Lagrangian for all interactions can be taken to be Hermitian. As a result, the couplings and form factors contributing to $\Delta \bar{\sigma}$ would be complex conjugates of those contributing to $\Delta \sigma$. Hence the real parts of these couplings would be equal, and the imaginary parts equal in magnitude, and opposite in sign. Thus, for a term labelled as $\mathrm{CP}$ even in one of the tables, the combination $\Delta \sigma^{-}$would be CP odd, and would survive only provided it comes with the imaginary part of couplings. For a term labelled CP odd, the combination $\Delta \sigma^{+}$would be $\mathrm{CP}$ odd, and would survive provided the corresponding couplings came with their real part. Thus, for example, in the $V, A$ case, the only surviving $\mathrm{CP}$ odd combinations correspond to the last rows in Tables 3 and 4, and the corresponding combination of couplings would be $\operatorname{Im}\left[\left(g_{V}^{e} g_{A}-g_{A}^{e} g_{V}\right) W_{2}\right]$. In the $S, P$ and $T$ cases, more possibilities survive, and we do not list them here.

\section{$5 \quad$ Processes with $t$-channel and $u$-channel exchanges}

So far we have dealt with a scenario where the SM interactions take place through $s$-channel $\gamma$ and $Z$ exchanges. This is most suitable for production of particles which have not direct coupling to $e^{-}$and $e^{+}$. However, for production of gauge bosons in the SM, which couple directly to $e^{+} e^{-}$, there would be a $t$-channel and/or $u$-channel lepton exchange.

It was also assumed that the new physics processes can also be represented by the $s$ channel exchange of a new particle, or by contact interactions.

The considerations of the early treatment could be carried over to these cases if one rewrites $t$ - and $u$-channel interactions or amplitudes as effective interactions, where the corresponding couplings, or rather form factors, depend on $t$ or $u$. Even in this case we have to deal with this new situation where the correlations obtained in the foregoing would get modified because of the $t$ and $u$ dependence of the form factors.

We first study how our discussion of the separate cases of $S, P, V, A$ and $T$ couplings can be adapted to this case. The crucial factor in this adaptation is the fact that for $m_{e}=0$, the only contributions which survive correspond to opposite $e^{-}$and $e^{+}$helicities. For, any finalstate particles which may be emitted from an electron line with a flip of electron helicity (as for example a Higgs boson) will have vanishing coupling in the limit of $m_{e}=0$. We are thus left in the most general case with only chirality-conserving combinations of Dirac matrices, 
sandwiched between electron and positron spinors of opposite helicities. Such a combination of Dirac matrices is a product of odd number of them. For massless spinors, they can always be reduced to a linear combination of $\gamma_{\mu}$ and $\gamma_{\mu} \gamma_{5}$. We are thus back to the case of $V$ and $A$ couplings in the $s$ channel considered in the preceding, except that the coefficients $g_{V}^{e}$ and $g_{A}^{e}$ would now be replaced by something more complicated. In fact, they could contain tensors constructed out of momenta occurring in the process. It is possible to absorb these tensors into the "hadronic" vertices. The final result would be that we could still use the tables we have obtained so far, with appropriate redefinitions of $g_{V}^{e}, g_{A}^{e}$ and the form factors. While this is a general feature, it is a discussion in very general terms not possibl. We will, instead, illustrate this for a special case of the process $e^{+} e^{-} \rightarrow \gamma Z$, which occurs in the SM through $t$ - and $u$-channel exchange of an electron.

The matrix element for the process $e^{-}\left(p_{-}\right)+e^{+}\left(p_{+}\right) \rightarrow \gamma_{\alpha}\left(k_{1}\right)+Z_{\beta}\left(k_{2}\right)$ can be written as

$$
M=e \bar{v}\left(p_{+}\right)\left[\frac{1}{t} \gamma^{\beta}\left(g_{V}^{e}-g_{A}^{e} \gamma_{5}\right)\left(\not p_{-}-\not p_{1}\right) \gamma^{\alpha}+\frac{1}{u} \gamma^{\alpha}\left(\not p_{-}-\not p_{2}\right) \gamma^{\beta}\left(g_{V}^{e}-g_{A}^{e} \gamma_{5}\right)\right] u\left(p_{-}\right),
$$

where $t=\left(p_{-}-k_{1}\right)^{2}$ and $u=\left(p_{-}-k_{2}\right)^{2}$. After some algebra, the equation above can be rewritten as

$$
M=e \bar{v}\left(p_{+}\right)\left[\gamma_{\mu}\left(g_{V}^{e}-g_{A}^{e} \gamma_{5}\right) T_{1}^{\mu \alpha \beta}+\gamma_{\mu}\left(g_{A}^{e}-g_{V}^{e} \gamma_{5}\right) T_{2}^{\mu \alpha \beta}\right] u\left(p_{-}\right)
$$

where

$$
T_{1}^{\mu \alpha \beta}=g^{\mu \beta}\left(\frac{2 p_{-}^{\alpha}}{t}-\frac{2 p_{+}^{\alpha}}{u}\right)+\left(-g^{\mu \alpha} k_{1}^{\beta}+g^{\alpha \beta} k_{1}^{\mu}\right)\left(\frac{1}{t}-\frac{1}{u}\right)
$$

and

$$
T_{2}^{\mu \alpha \beta}=-i \epsilon^{\mu \alpha \beta \lambda} k_{1 \lambda}\left(\frac{1}{t}+\frac{1}{u}\right) .
$$

As can be seen from the above, we are in a position to use the formalism of the previous sections, except that the SM contribution has a different form for the "hadronic" vertex, while the leptonic vertex remains of the $V$ and $A$ form. We could thus go ahead and consider the cross term of this with the new interactions. So far as the leptonic tensor concerned, we only have to modify what we use for $g_{V}^{e}$ and $g_{A}^{e}$. The hadronic tensor would now be more complicated and would have to be computed using the cross term of $T_{1}$ and $T_{2}$ above with the tensors arising in the BSM interactions. There is an apparent problem in that the "hadronic" tensor will involve leptonic momenta, as for example, in $T_{1}$. However, on summing over final state polarizations, the leptonic momenta will be contracted appropriately to give Lorentz scalars like $p_{-} . k_{1}, p_{-} . k_{2}$, etc., which can be rewritten in terms of $s, t$ and $u$, and will contribute to the $t$ - and $u$-dependence of the effective new form factors.

The next important issue we have to deal with, then, is the $t$ and $u$ dependence of form factors, which was assumed absent in the simple treatment which led to the Tables 1-6. If we treat processes involving $t$ - and $u$-channel exchanges, the propagators corresponding to these exchanges will contribute to the $t$ and $u$ dependence of the form factors. 
In the original inclusive process (11) which we consider, the kinematic variables $t$ and $u$ may be written as

$$
t=\left(p_{-}-p\right)^{2}=-2 p_{-} \cdot p+p^{2}, u=\left(p_{+}-p\right)^{2}=-2 p_{+} \cdot p+p^{2}
$$

Since $t+u=-2 p \cdot q+2 p^{2}$, it is only the dependence on the combination $t-u=4 \vec{p} \cdot \vec{K}$ which is new when we allow form factors to depend on $t$ and $u$. Thus, in such a case, the correlations of Tables 1-6 would get additional dependence on $\vec{p} \cdot \vec{K}$. However, they would not get any additional dependence on triple product of vectors, or on spin. This dependence on $\vec{p} \cdot \vec{K}$ would also have important consequences when we discuss the CP properties of the correlations.

In the case when $H=\bar{H}$, a CP transformation simply interchanges $t$ and $u$. Then the $\mathrm{CP}$ behaviour of a certain correlation is even or odd depending on the product of the CP phase factor obtained from the relevant table and a factor \pm 1 coming from the behaviour of the amplitude under $t$ - $u$ interchange.

In the example of the process $e^{+} e^{-} \rightarrow \gamma Z$ considered above, the form factor $T_{1}$ is odd under the interchange of $t$ and $u$. When the new interactions are CP violating, it could give rise to a CP-odd correlation of one of the forms listed in Tables 3 and 4 as being CP even. $T_{1}$ is proportional to $(t-u)$, and the consequent factor of $\vec{K} \cdot \vec{p}$, as it turns out, multiplies the last entry in Table 4, giving rise to a CP-odd correlation when the new interaction considered is either an anomalous $\gamma \gamma Z$ coupling or a contact $e^{+} e^{-} \gamma Z$ interaction. We refer to [8, 9] for details.

The same CP-odd correlation arises in a different context of supersymmetry in the process

$e^{+} e^{-} \rightarrow \tilde{\chi}_{i} \tilde{\chi}_{j}, i \neq j$, where $\tilde{\chi}_{i}$ are neutralinos in the theory, which are self-conjugate. Here the CP-odd terms arise in the cross terms between the $s$-channel and the $t$ - and $u$-channel production diagrams [16, 17.

In the case when $H \neq \bar{H}$, and the final state is $H \bar{H}$, a CP transformation keeps $t$ and $u$ unchanged. Hence the earlier discussion of CP properties of correlations for this case goes through unchanged.

In the case of a final state $H \bar{H}^{\prime}\left(H \neq H^{\prime}\right)$, one would compare the cross section combinations $\Delta \sigma_{ \pm}$, which are linear combinations of cross sections for the production of $H \bar{H}^{\prime}$ and $\bar{H} H^{\prime}$. In this case, under CP, $t$ and $u$ for the first process would go respectively to $t$ and $u$ for the second process. Thus, the discussion of CP properties given earlier for this case goes through. The specific example of chargino pair production in supersymmetry would serve as an illustration for this case, which is discussed in the next section.

\section{Some applications}

In our earlier work on $e^{+} e^{-} \rightarrow t \bar{t}[3]$ we had considered the possibility of CP violation arising from four-Fermi interactions due to an effective Lagrangian framework with transversely polarized beams. We found that there was no CP-violating observable possible with $V$ and 
$A$ type of four-Fermi interactions. However, CP-violating observables did exist for $S, P$ and $T$ interactions. The negative result for $V, A$ interactions is clear from our discussion of the case 2 a of Sec. 4. For the $S, P$ and $T$ case, it is possible to have CP-odd observables, and of the possible ones listed therein, the ones which occur in the special case of lowestdimensional observables are $\operatorname{Re}\left(g_{P} F\right)$ and $\operatorname{Re}\left(g_{T} F_{2}\right)$, corresponding respectively to the fourFermi couplings $\operatorname{Im}\left(S_{R R}\right)$ and $\operatorname{Im}\left(T_{R R}\right)$ in the notation of [3]. The special features observed in that work, viz., that the four-Fermi scalar coupling terms occur with only the $g_{A}^{e}$ coupling at the electron vertex, and that the tensor coupling terms occur with only the $g_{V}^{e}$ at the electron vertex, are borne out by our general results.

Rizzo [12] has considered probing extra-dimensional models using transverse polarization. The key observation is that an azimuthal angle dependence of the form $\cos 2 \phi$ and $\sin 2 \phi$ enters the differential cross section for fermion pair production in $e^{+} e^{-}$collisions, when the $s$-channel exchange of a tower of massive gravitons is introduced. Here $\phi$ is the azimuthal angle of the fermion defined relative to the $e^{-}$beam axis as the $x$ axis, and the $e^{-}$transverse polarization direction as the $y$ axis. The coupling of gravitons at the leptonic vertex has the form

$$
\Gamma_{\mu \nu}=\gamma_{\mu} q_{\nu}+\gamma_{\nu} q_{\mu}
$$

So far as the Dirac structure is concerned, there is only one $\gamma$ matrix in each term, and so this falls in the category of vector interactions. The four-vector $q_{\nu}$ appearing in the coupling could be absorbed in the hadronic tensor. Thus, we could use the results of the third and seventh rows of Table 3 to deduce the $\theta$ and $\phi$ dependence of the angular distribution for any final state.

In a recent work [18, the authors have considered the possibility of studying noncommutative extension of the standard model through $Z \gamma$ production at the Tevatron and the LHC. It would be possible to extend this discussion to the possibility of probing an underlying non-commutative field theory at the linear collider with polarized beams. They note that to leading order in the non-commutativity parameter, contact interactions as well as triple-gauge couplings are introduced to describe the process $f \bar{f} \rightarrow Z \gamma$. The technique developed here would be useful to study angular distributions for the case of $e^{+} e^{-} \rightarrow Z \gamma$ in this model.

We now apply an extension of our results to two examples where the underlying theory is not the SM, but the MSSM. The first example is of neutralino pair production, already alluded to in Sec. 4. In this case there is an $s$-channel as well as $t$ - and $u$-channel contributions. The $t$ - and $u$-channel contributions can be written using Fierz transformation in a form which has only $V$ and $A$ couplings to the electron current [16], but with an overall factor proportional to $(t-u)$ coming from the propagators. We can then apply our results obtained for $V, A$ BSM interactions from Table 3 and 4 . The case 1 considered in Sec. 4 for self-conjugate particles applies in this case. We then find that it is possible to have a $\mathrm{CP}$-odd correlation given by a product of the correlation in the last line of either Table 3 or 4 and the factor $\vec{p} \cdot \vec{K}$, where $p$ is the momentum of a neutralino. This has been discussed in ref. [16, 17] and a numerical study of a corresponding CP-odd asymmetry has been carried 
out in ref. [17.

The other example from MSSM is that of chargino pair production. This corresponds to the case $2 \mathrm{~b}$ discussed in Sec. 4. We also refer the reader to the comments in Sec. 5 regarding the $\mathrm{C}$ transformation of the propagators entering the amplitude in this case. We find that for the process $e^{+} e^{-} \rightarrow \tilde{\chi}_{i}^{+} \tilde{\chi}_{j}^{-}$, where $\tilde{\chi}_{i}^{ \pm}$denote charginos, no CP-odd correlation exists at tree level [19]. This can be understood from our discussion earlier where we found that in the $V, A$ case, to which our present case can be reduced using a Fierz transformation, the only CP-odd correlation arises from the last lines in Tables 3 and 4 , in the combination $\Delta \sigma_{-}$ corresponding to the difference of the cross sections for $e^{+} e^{-} \rightarrow \tilde{\chi}_{i}^{+} \tilde{\chi}_{j}^{-}$and $e^{+} e^{-} \rightarrow \tilde{\chi}_{j}^{+} \tilde{\chi}_{i}^{-}$. However, it is found in [19] that the corresponding coefficient vanishes at tree level.

Another example from the MSSM which would fall in the category of case $2 \mathrm{~b}$ of Sec. 4 is the neutralino pair-production process mentioned above, but when the energy and momentum that is measured is of a lepton arising in the decay chain of a neutralino. In this case, it is possible to construct a CP-odd correlation using leptons of opposite charges, and the effect is non-vanishing [17].

\section{Conclusions}

To conclude, we have considered the exploration of the space-time structure of new physics beyond the standard model in polarized $e^{+} e^{-}$annihilation at linear collider energies. We have studied the interference of the standard model processes for a final state whose momentum alone is measured and have considered the most general possible polarization of the electron and positron beams. Our work is the analog of the exploration of the space-time structure of the neutral current due to the $Z$ boson from its interference with the QED amplitude for a given final state. While our work is a logical extension of the work of Dass and Ross, it has several novel features which were not present in that work. We have provided a significant extension not precedented in the literature in our discussion of the case of SM amplitudes which occur with fermion exchange in the $t$ and $u$ channels, by showing that they may be written in a form analogous to those with $s$-channel amplitudes, albeit with momentum dependent form factors. We have also shown that some features of our treatment can be carried over to an extension of SM, like MSSM, using as illustrations chargino and neutralino pair production. We have also considered popular scenarios for BSM physics, resulting from either extra dimensional models or from non-commutative models.

\section{Acknowledgements}

We thank the organizers and sponsors (BRNS) of WHEPP8 (Workshop on High Energy Physics Phenomenology 8) held at IIT Mumbai in January 2004, where this work was initiated. We thank Alfred Bartl for a careful reading of the manuscript and useful suggestions. BA thanks N.D. Hari Dass for a discussion. BA also thanks the Council for Scientific and In- 
dustrial Research for support during the course of these investigations under scheme number 03(0994)/04/EMR-II, as well as the Department of Science and Technology, Government of India.

\section{References}

[1] T. Abe et al. [American Linear Collider Working Group], in Proc. of the $A P S / D P F / D P B$ Summer Study on the Future of Particle Physics (Snowmass 2001) ed. N. Graf, arXiv:hep-ex/0106055.

J. A. Aguilar-Saavedra et al. [ECFA/DESY LC Physics Working Group], arXiv:hep-ph/0106315.

K. Abe et al. [ACFA Linear Collider Working Group], arXiv:hep-ph/0109166.

K. Ackermann et al., DESY-PROC-2004-01 Prepared for 4th ECFA / DESY Workshop on Physics and Detectors for a 90-GeV to 800-GeV Linear e+e-Collider, Amsterdam, The Netherlands, 1- 4 Apr 2003

[2] G. Moortgat-Pick et al., arXiv:hep-ph/0507011.

P. Osland and N. Paver, arXiv:hep-ph/0507185.

[3] B. Ananthanarayan and S. D. Rindani, Phys. Rev. D 70, 036005 (2004) arXiv:hep-ph/0309260.

[4] S. D. Rindani, Pramana 61, 33 (2003) arXiv:hep-ph/0304046.

[5] B. Ananthanarayan, S. D. Rindani and A. Stahl, Eur. Phys. J. C 27, 33 (2003) arXiv:hep-ph/0204233.

[6] B. Ananthanarayan and S. D. Rindani, Phys. Rev. Lett. 73, 1215 (1994) arXiv:hep-ph/9310312.

B. Ananthanarayan and S. D. Rindani, Phys. Rev. D 50, 4447 (1994) arXiv:hep-ph/9403346.

B. Ananthanarayan and S. D. Rindani, Phys. Rev. D 51, 5996 (1995) arXiv:hep-ph/9411399.

[7] D. Choudhury and S. D. Rindani, Phys. Lett. B 335, 198 (1994) arXiv:hep-ph/9405242.

[8] B. Ananthanarayan, S. D. Rindani, R. K. Singh and A. Bartl, Phys. Lett. B 593, 95 (2004) [Erratum-ibid. B 608, 274 (2005)] arXiv:hep-ph/0404106.

[9] B. Ananthanarayan and S. D. Rindani, Phys. Lett. B 606, 107 (2005) arXiv:hep-ph/0410084. 
[10] B. Ananthanarayan and S. D. Rindani, JHEP 0510, $077 \quad$ (2005) arXiv:hep-ph/0507037.

[11] K. i. Hikasa, Phys. Rev. D 33, 3203 (1986).

J. Fleischer, K. Kolodziej and F. Jegerlehner, Phys. Rev. D 49, 2174 (1994).

M. Diehl, O. Nachtmann and F. Nagel, Eur. Phys. J. C 32, 17 (2003) arXiv:hep-ph/0306247.

[12] T. G. Rizzo, JHEP 0302, 008 (2003) arXiv:hep-ph/0211374.

T. G. Rizzo, JHEP 0308, 051 (2003) arXiv:hep-ph/0306283.

[13] G. V. Dass and G. G. Ross, Phys. Lett. B 57, 173 (1975).

[14] G. V. Dass and G. G. Ross, Nucl. Phys. B 118, 284 (1977).

[15] B. Grzadkowski, Acta Phys. Polon. B 27, 921 (1996) arXiv:hep-ph/9511279.

B. Grzadkowski, Z. Hioki and M. Szafranski, Phys. Rev. D 58, 035002 (1998) arXiv:hep-ph/9712357.

[16] S. Y. Choi, J. Kalinowski, G. Moortgat-Pick and P. M. Zerwas, Eur. Phys. J. C 22, 563 (2001) [Addendum-ibid. C 23, 769 (2002)] arXiv:hep-ph/0108117.

[17] A. Bartl, H. Fraas, S. Hesselbach, K. Hohenwarter-Sodek, T. Kernreiter and G. Moortgat-Pick, arXiv:hep-ph/0510029.

[18] A. Alboteanu, T. Ohl and R. Ruckl, arXiv:hep-ph/0511188.

[19] A. Bartl, K. Hohenwarter-Sodek, T. Kernreiter and H. Rud, Eur. Phys. J. C 36, 515 (2004) arXiv:hep-ph/0403265. 